Dimroth und Scheurer, Uber den Farbstoff des Kermes. 43

Zum Unterschiede vom 2,6-Dioxynaphthochinon löst sich diese Substanz in Natronlauge und ebenso in konz. Schwefelsäure mit karmoisinroter Farbe. Die Lösungen gaben keine charakteristischen Absorptionsstreifen.

Bromierung des 2,7-Dioxynaphthochinons.

Bei dieser Substanz gelingt es, in einer Operation drei Bromatome einzuführen. 0,5 $\mathrm{g}$ wurden in Eisessiglösung mit der 10 fachen Menge Brom, das man in Portionen zufügt, 7 Stunden am Rückflußkühler gekocht. Beim Erkalten erfolgt langsam Krystallisation. Tribrom2,7-dioxynaphthochinon krystallisiert aus Eisessig in hellgelben Prismen rom Schmelzp. 228-229 ${ }^{\circ}$, die sich in Soda und Natronlauge mit viel blaustichigerer Farbe lösen als das Isomere, in konz. Schwefelsäure mit braunroter Farbe, die in dünner Schicht ins Olive spielt.

$\begin{array}{ccr}0,2124 \mathrm{~g} \text { gaben } & 0,2208 \mathrm{CO}_{2} \text { und } 0,0178 \mathrm{H}_{2} \mathrm{O} . \\ 0,2357 \mathrm{~g} & 0,3117 \mathrm{AgBr}^{2} & \\ & \text { Ber. für } \mathrm{C}_{10} \mathrm{H}_{8} \mathrm{O}_{4} \mathrm{Br}_{3} & \text { Gef. } \\ \mathrm{C} & 28,11 & 28,35 \\ \mathrm{H} & 0,71 & 0,94 \\ \mathrm{Br} & 56,19 & 56,28\end{array}$

\title{
III. Über den Farbstoff des Kermes;
}

von Otto Dimroth und Wilhelm Scheurer.

Das Hauptresultat der ersten Studie, welche der eine von uns über den Farbstoff der Kermesschildläuse veröffentlicht hat ${ }^{1}$ ), war die Konstatierung der nahen strukturellen Beziehungen, welche zwischen diesem Farbstoff und der Carminsäure bestehen. Kermessäure gab dieselben Spaltstücke wie Carminsäure, nämlich Nitrococcussäure und Cochenillesäure. Da das Molekül der

1) Ber. d. d. chem. Ges. 43, 1387 (1910). 
Kermessäure erheblich einfacher gebaut ist als das seines berühmteren Rivalen, durfte man hoffen, rascher einen Einblick in den Bau dieses Farbstoffes zu bekommen und so indirekt auch die Kenntnis der Carminsäure zu fördern. Diese Voraussetzung hat sich erfüllt, die Konstitution der Kermessäure kann jetzt als in allen wesentlichen Punkten festgelegt gelten.

Die größere Menge an Ausgangsmaterial, welche wir zu unseren Versuchen brauchten, gab Gelegenheit, die Reindarstellung des Farbstoffes noch sorgfältiger auszuarbeiten. Dabei wurde gefunden, dab in der Rohkermessäure ein zweiter schön krystallisierender Farbstoff vorhanden ist, den wir Flavokermessäure nennen wollen. $\mathrm{Da}$ er in seinen färberischen Eigenschaften ganz und gar von der Kermessäure abweicht, also vermutlich nicht zur Gruppe der Carminsäurefarbstoffe gehört, und da nur sehr geringe Mengen zur Verfügung standen, haben wir uns damit begnügt, seine Zusammensetzung festzustellen, ohne ihn näher zu untersuchen.

Für die Aufklärung der Konstitution der Kermessäure hat das Studium des Verhaltens gegen Brom wesentliche Dienste geleistet. Bromiert man in siedender 50 prozentiger Essigsäure in der Hitze, so erhält man als Hauptreaktionsprodukt $\alpha$-Bromcarmin,<smiles>CC1C(Br)C(O)C(Br)C2C(=O)C(Br)C(Br)C12</smiles>

das unter diesen Bedingungen auch aus der Carminsäure erhalten wird.

Viel weniger weit wird das Molekül abgebant, wenn man Brom in wasserfreier siedender Essigsäure auf Kermessäure reagieren läßt. Man erhält dann in recht glatter Reaktion einen Farbstoff, der in seinem Gesamtverhalten der Kermessäure noch sehr nahe steht, 
in seiner Zasammensetzung aber sich dadurch unterscheidet, daß er 2 At. Kohlenstoff im Molekül weniger enthält. Die Formel der Kermessäure ist $\mathrm{C}_{18} \mathrm{H}_{12} \mathrm{O}_{9}$, die der neuen Substanz, die wir Monobromcoccin nennen. wollen, $\mathrm{C}_{16} \mathrm{H}_{9} \mathrm{BrO}_{8}$.

Monobromeoccin enthält die Carboxylgruppe, die in der Kermessäure vorhanden ist, noch unverändert, und ebenso wie dieser Farbstoff vier phenolische Hydroxylgruppen. Bei der Oxydation mit Wasserstoffsuperoxyd in alkalischer Lösung erhält man Cochenillesäure.<smiles>CC1CC(O)C(C(=O)O)C(C(=O)O)C1C(=O)O</smiles>

Da man nicht daran zweifeln kann, dab Kermessäure und Bromcoccin wie die Carminsäure den Naphthochinonring enthalten, kommt man za dem Schluß, dab. in dem Bromcoccin die Gruppierung<smiles>CC(OC(=O)O)C1CC(O)CC(C(=O)O)C2C(=O)C=CC(=O)C12</smiles>

vorliegt. Die vier Kohlenstoffatome mit den vier einwertigen Gruppen lassen sich aber nur in einem Ringsystem nnterbringen. Aus ganz anderen Gründen wie bei der Carminsäure gelangt man auch hier zu der Schlußfolgerung, dab das Bromcoccin und dann natürlich auch die Kermessäure Abkömmlinge des Anthrachinons ${ }^{1}$ ) sind.

Für die Verteilung der drei Hydroxylgruppen und des Bromatoms im Ring existieren vier Möglichkeiten. Die zwei Formeln mit drei vicinalen Hydroxylgruppen

1) Läge das Ringsystem des Phenanthrenchinons vor, so. müBte man durch Oxydation eine substituierte Diphensäure, nicht. Cochenillesäure, erhalten. 
sind auszuschließen, denn dann wäre das Bromcoccin ein Substitutionsprodukt des Anthragallols, von dem es in seinem färberischen Verhalten gänzlich abweicht. Dagegen steht es dem Purpurin nahe, so dab nur die Wahl bleibt zwischen zwei Strukturformeln<smiles>CC1CC(O)C(C(=O)O)C2C(=O)C3C(O)C(Br)C(O)C(O)C3C(=O)C12</smiles>

und<smiles>CC1CC(O)C(C(=O)O)C2C(=O)C3C(O)C(O)C(Br)C(O)C3C(=O)C12</smiles>

Diese Frage, die übrigens von relativ untergeordneter Bedeutung ist, ist noch unentschieden. Wichtiger ist die andere Frage, welchen Rückschlub man nun von der Konstitution des Bromcoccins auf die der Kermessäure ziehen kann.

Der Vergleich der empirischen Formeln lehrt, daß im Bromcoccin ein Bromatom an Stelle der Gruppe $\mathrm{C}_{2} \mathrm{H}_{3} \mathrm{O}$ eingetreten ist, die überaus grobe Ähnlichkeit der beiden Farbstoffe - die Spektren der alkalischen Lösungen sind identisch, diejenigen der schwefelsauren Lösungen ganz nahe verwandt - weist zwingend darauf hin, daß dieser Ersatz ohne wesentliche Veränderung des Gesamtmoleküls vor sich gegangen ist.

Der Rest $\mathrm{C}_{2} \mathrm{H}_{3} \mathrm{O}$ kann nur als $-\mathrm{CH}_{2}-\mathrm{C} \ll \mathrm{H}$ oder - $\mathrm{CO}-\mathrm{CH}_{3}$ formuliert werden. Das erstere ist unwahrscheinlich, denn die Kermessäure hat nicht die Eigenschaften eines Aldehyds; auch wäre nicht zu verstehen, daß dieser Rest durch Brom sollte verdrängt werden. Daß dagegen die Acetylgruppe durch Brom eliminiert wird, ist nicht ohne Analogien und diesem Fall besonders plausibel, wenn man berïcksichtigt, daß in der Purpurincarbonsäure<smiles>O=C(O)C1C(O)C(O)C2C(=O)C3CCCCC3C(=O)C2C1O</smiles> 
die Carboxylgruppe sehr locker sitzt und schon durch siedendes Wasser abgesprengt wird. Es ist durchaus zu erwarten, daß die Acetylgruppe an derselben Stelle zwar fester als Carboxyl, aber immerhin ziemlich beweglich eingefügt sein wird.

Das Ergebnis dieser Versuche und Überlegungen ist also, daß die Kermessäure eine der folgenden Strukturformeln besitzt:<smiles>COC(O)C1C(O)C(O)C2C(=O)C3C(C)CC(O)C(C(=O)O)C3C(=O)C2C1O</smiles><smiles>CC(=O)C1C(O)C(O)C2C(=O)C3C(C(=O)C2C1O)C(C)CC(O)C3C(=O)O</smiles>

Bromiert man das Monobromcoccin in methylalkoholischer Lösung weiter, so wird die Carboxylgruppe eliminiert und noch ein Wasserstoffatom durch Brom ersetzt. Für dies „Tribromcoccin" stehen die Strukturbilder<smiles>CC1C(Br)C(O)C(Br)C2C(=O)C3C(O)C(Br)C(O)C(O)C3C(=O)C12</smiles><smiles>CC1C(Br)C(O)C(Br)C2C(=O)C3C(O)C(O)C(Br)C(O)C3C(=O)C12</smiles>

zur Auswahl.

Die Ansicht, daß die Kermessäure wie die Carminsäure ein Anthrachinonderivat ist, erhält eine kräftige Stütze durch das Ergebnis der Zinkstaubdestillation. Man erhält dabei in allerdings schlechter Ausbeute da die Kermessäure nicht unzersetzt destilliert, sondern beim Erhitzen verkohlt - ein Gemenge von Kohlenwasserstoften, aus dem sich $\alpha$-Methylarthracen isolieren und in Form seines Chinons identifizieren lieb. Daneben entsteht vermutlich Anthracen, das jedoch nicht in ganz reinem Zustande gefaßt werden konnte. 


\section{Experimenteller Teil. \\ Darstellung der Kermessäure und Flavokermessäure.}

Die in der ersten Mitteilung ausführlich beschriebene Darstellungsmethode wurde im wesentlichen beibehalten. Wie damals erwähnt wurde, besitzt diejenige Portion des Farbstoffes, welche aus der Ätherlösung durch Ausschü̈teln mit Natriumacetat gewonnen wird, einen geringeren Grad der Reinheit als der andere Teil, der aus dem Äther anskrystallisiert. Diese Verunreinigung besteht nun, wie sich neuerdings herausstellte, aus einem zweiten, gut krystallisierenden Farbstoff, welchen wir Flavokermessäure nennen wollen.

Es ist leicht zu erkennen, ob einem Präparat von Kermessäure etwas von diesem Farbstoff beigemengt ist. Reine Kermessäure löst sich nämlich in konzentrierter Schwefelsäure auf Zusatz von Borsäure mit rein blauer Farbe; enthält sie dagegen Flavokermessäure, so ist die Farbe stumpf und bei Anwesenheit gröberer Mengen schmutzig blauviolett.

Zur Trennung eignet sich die verschiedene Löslichkeit der Natriumsalze. Das Dinatriumsalz der Kermessäure ist in heiber wäbriger, doppelt normaler Natriumacetatlösung fast unlöslich, das Natriumsalz der Flavokermessäure dagegen ziemlich leicht löslich.

Man verfährt wie folgt: Das Gemisch der Farbstoffe wird in heißem Alkohol gelöst; dazu gibt man wäßriges Natriumacetat erst tropfenweise unter Umschütteln und fügt schließlich, wenn die Kermessäure völlig ausgefällt ist, noch einen sehr großen Überschuß von Natriamacetat $z u$, erwärmt noch eine halbe Stunde anf dem Wasserbad, filtriert durch den Dampftrichter und wäscht wiederholt mit heißer Natriumacetatlösung nach. Die Waschwässer werden getrennt aufgefangen.

Aus dem un\}öslichen dunkeln Natrinusal\% erhält man die freie hermes.äure, indem man 's in heißem Wasser unter Zusutz von Soda auflöst und dann unter Umrühren in heiße verdünnte Salzsäure gießt. 
Nach dem Erkalten saugt man die Kermessäure ab, prüft sie mit Schwefelsäure-Borsäure auf Reinheit und wiederholt nötigenfalls das Verfahren.

Aus der heißen Natriumacetatlösung krystallisiert beim Erkalten das Natriumsalz der Flavokermessäure in gelbbraunen Krystallen aus, meist noch mit etwas Kermessäure verunreinigt. Man setzt die Flavokermessäure in Freiheit und verwandelt sie anf dieselbe Weise ein zweites Mal in das Natriumsalz.

Die Menge der Flavokermessäure ist sehr gering; aus $5 \mathrm{~kg}$ Kermes, erhält man etwa $3 \mathrm{~g}$ dieses Farbstoffes, den wir deshalb vorläufig nicht eingehender untersucht haben.

Flavokermessäure krystallisiert aus Alkohol in langen der Kermessäure sehr ähnlichen Nadeln, aus wasserhaltiger Essigsäure in breiten durchkreuzten Prismen. In der Löslichkeit steht sie der Kermessäure sehr nahe, ist jedoch in allen Solvenzien etwas leichter löslich.

In kaltem Wasser löst sie sich sehr schwer mit braungelber Farbe, die Lösung in konz. Schwefelsänre ist rotbraun, auf Zusatz von Borsäure zeigt sie keinen wesentlichen Farbenumschlag. Die Farbe in Natronlange ist der Kermessäure ähnlich, doch mehr braunstichig. Die Spektren der alkalischen und der schwefelsauren Lösung zeigen durchaus keine Ähnlichkeit mit der Kermessäure, sie weisen keine Absorptionsstreifen auf, sondern zeigen allgemeine, vom violetten Ende ausgehende Absorption.

Zur Analyse wurden Präparate verwendet, die mehrfach ans Essigsäure umkrystallisiert und im Vakuum der Quecksilberpumpe bei $100^{\circ}$ zur Gewichtskonstanz getrocknet waren. Die einfachste Formel, die sich aus der Analyse berechnen läßt, ist $\mathrm{C}_{13} \mathrm{H}_{8} \mathrm{O}_{8}$.

$0,2100 \mathrm{~g}$ gaben $0,4620 \mathrm{CO}_{2}$ und $0,0606 \mathrm{H}_{2} \mathrm{O}$.

$0,2257 \mathrm{~g} \quad " \quad 0,4959 \quad \mathrm{CO}_{2} \quad, \quad 0,0677 \mathrm{H}_{2} \mathrm{O}$.

$0,2764 \mathrm{~g} \quad " \quad 0,6077 \mathrm{CO}_{2} \quad, \quad 0,0809 \mathrm{H}_{2} \mathrm{O}$.

Anualen der Chemie 399. Band. 


\begin{tabular}{lcrrr} 
& Ber. für $\mathrm{C}_{18} \mathrm{H}_{8} \mathrm{O}_{6}$ & \multicolumn{3}{c}{ Gef. } \\
$\mathbf{C}$ & 60,00 & $\mathbf{6 0 , 0 0}$ & $\mathbf{5 9 , 7 1}$ & $\mathbf{5 9 , 9 6}$ \\
$\mathbf{H}$ & 3,10 & $\mathbf{3 , 2 3}$ & $\mathbf{3 , 3 4}$ & $\mathbf{3 , 2 7}$
\end{tabular}

Flavokermessänre färbt ungebeizte Wolle aus saurem Bade goldgelb an, ohne das Bad zu erschöpfen; auf Tonerdebeize gibt sie ein fahles Braun, auf Zinn ein stumpfes Orange.

\section{Salze der Kermessäure.}

Unsere frühere Mitteilung ist noch durch einen Versuch zu ergänzen, welcher zeigt, daß die Kermessäure im Maximum fünf Äquivalente Metall zu binden vermag.

Zn einem starken Überschub titrierten Barytwassers, der sich in einem Meßkolben befindet, gibt man aus einem Tropftrichter eine alkoholische Lösung von Kermessäure unter lebhaftem Umschütteln, spült mit Alkohol nach und digeriert einige Zeit auf dem Wasserbad. Nach dem Erkalten füllt man zur Marke auf, läßt absitzen und filtriert unter AbschluB von Kohlensäure. Ein aliquoter Teil der Lösung wird alsdann zurücktitriert. Das Bariumsalz der Kermessäure ist in Wasser nahezu völlig unlöslich.

$0,1952 \mathrm{~g}$ Kermessäure verbrauchten $27,1 \mathrm{ccm} \mathrm{n} / \mathrm{1}^{-}$-Barytwasser. $0,2008 \mathrm{~g} \quad " \quad 27,6 \mathrm{ccm} \mathrm{n/10^{- }}$

Für 5 Äquivalente berechnet 26,3 und $27,0 \mathrm{ccm}$.

Das in der vorigen Mitteilung beschriebene saure Bariumsalz, von welchem seinerzeit nur eine Bariumbestimmung ausgeführt worden war, wurde nochmals vollständig analysiert.

$$
\begin{aligned}
& 0,2260 \mathrm{~g} \text { gaben } 0,4100 \mathrm{CO}_{2} \text { und } 0,0546 \mathrm{H}_{2} \mathrm{O} . \\
& 0,2574 \mathrm{~g}, \quad 0,4612 \mathrm{CO}_{2}, 0,0590 \mathrm{H}_{2} \mathrm{O} \text {. } \\
& 0,2674 \mathrm{~g} \quad, \quad 0,0720 \mathrm{BaSO}_{4} \text {. } \\
& \begin{array}{lcrr} 
& \text { Ber. für }\left(\mathrm{C}_{18} \mathrm{H}_{11} \mathrm{O}_{9}\right)_{2} \mathrm{Ba} & \multicolumn{2}{c}{\text { Gef. }} \\
\mathrm{C} & 49,11 & 49,48 & 48,87 \\
\mathrm{H} & 2,52 & 2,70 & 2,56 \\
\mathrm{Ba} & 15,62 & 15,62 & 15,85
\end{array}
\end{aligned}
$$




\section{Decarboxykermessäure.}

$1 \mathrm{~g}$ Kermessänre wird mit $50 \mathrm{ccm}$ Wasser im EinschluBrohr 10-15 Stunden auf $150^{\circ}$ erhitzt. Beim öffnen des Rohres läßt sich Kohlensäure nachweisen. Das Rohr ist von langen dunkelroten Krystallnadeln erfüllt, die abgesaugt und aus 80 prozentiger Essigsäure umkrystallisiert werden. Die wäßrige Flüssigkeit gibt nach Zusatz von Salzsäure einen Niederschlag, der vorwiegend aus unveränderter Kermessäure besteht.

Decarboxykermessäure krystallisiert in roten Nädelchen von schönem Glanz, die dunkler gefärbt sind wie Kermessäure. In Wasser ist sie sehr schwer löslich, in Bicarbonat löst sie sich, da die Carboxylgruppe fehlt, fast gar nicht. In Natronlauge löst sie sich mit derselben Farbe wie Kermessäure; auch das Spektrum - Absorptionsstreifen bei $520-530$ und $560-580 \mu \mu$ - ist identisch. Die Farbe in konz. Schwefelsäure ist blauer als bei Kermessäure; auf Zusatz von Borsäure werden die Farben identisch. Die Schwefelsäurelösung zeigt einen verwaschenen Absorptionsstreifen bei 500-525, der auf Zusatz von Borsäure schärfer wird.

Im Schmelzpunktröhrchen erhitzt fängt die Decarboxysäure über $150^{\circ}$ an zu sublimieren, färbt sich von $250^{\circ}$ an dunkel und verkohlt, ohne zu schmelzen.

Zur Analyse wurde die Substanz im Hochvakuum bei $100^{\circ}$ getrocknet und mit Bleichromat verbrannt.

$$
\begin{aligned}
& 0,2282 \mathrm{~g} \text { gaben } 0,5229 \mathrm{CO}_{2} \text { und } 0,0737 \mathrm{H}_{2} \mathrm{O} \text {. }
\end{aligned}
$$

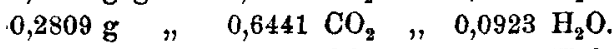

$$
\begin{aligned}
& 0,2179 \mathrm{~g} \quad, \quad 0,4992 \mathrm{CO}_{2} \quad \text { " } 0,0723 \mathrm{H}_{2} \mathrm{O} \text {. } \\
& \text { Ber. für } \mathrm{C}_{17} \mathrm{H}_{12} \mathrm{O}_{7} \\
& \begin{array}{lllll}
\mathrm{C} & 62,20 & 62,49 & 62,53 & 62,48
\end{array} \\
& \begin{array}{lllll}
H & 3,66 & 3,61 & 3,67 & 3,71
\end{array}
\end{aligned}
$$

Die Decarboxysäure färbt gebeizte $\mathrm{W}$ olle und Baumwolle mit denselben Tönen an wie Kermessäure, zieht jedoch aus saurem Bade nicht auf ungebeizte Wolle. 
Bromierung der Kermessäure.

Die Reaktionsbedingungen, insbesondere die Natur des Lösungsmittels, sind von außerordentlichem Einfluß bei der Bromierung der Kermessäure; im folgenden sollen nur diejenigen Versuche beschrieben werden, die einen möglichst einheitlichen Verlauf nahmen.

\section{Monobroncoccin.}

$1 \mathrm{~g}$ Kermessäure wird sehr fein verrieben in $50 \mathrm{ccm}$ wasserfreiem Eisessig suspendiert. Dazu gibt man $2 \mathrm{ccm}$ Brom und kocht 1-2 IIinuten. Der Farbstoff geht in Lösung; von etwa unangegriffenen Partikelchen filtriert man ab und läßt erkalten. Monobromcoccin krystallisiert in roten Prismen aus. Die Ausbeute beträgt etwa $1 \mathrm{~g}$. Unlöslich in Wasser, schwer löslich in Äther und Benzol, leicht in Alkohol. Krystallisiert aus verdünntem Alkohol in langen, feinen Nadeln.

In Natriumbicarbonat löst es sich unter Kohlensäureentwicklung mit stumpf kirschroter Farbe; in Natronlauge mit der Farbe der Kermessäure und identischem Spektrum; in Schwefelsänre mit rosenroter Farbe, die auf Zusatz von Borsäure in blauviolett umschlägt. Die Lösung in Schwefelsäure hat zwei Streifen im Grün bei $500-510$ und $540-550 \mu \mu$; die borsäurehaltige Lösung einen verwaschenen Streifen bei $525-535$ und bei 560 bis $575 \mu \mu$. Zur Analyse wurde bei $100^{\circ}$ im Vakuum getrocknet. Die getrocknete Substanz schmilzt bei 259 bis $260^{\circ}$ unter Zersetzung. Aus Eisessig krystallisiert der Farbstoff mit 2 Mol. Eisessig.

$0,8787 \mathrm{~g}$ verloren beim Trocknen $0,1942 \mathrm{~g}$ entspr. 22,10 Proz.

Berechnet 22,69 Proz.

$0,2011 \mathrm{~g}$ gaben $0,3445 \mathrm{CO}_{2}$ und $0,0395 \mathrm{H}_{2} \mathrm{O}$.

$0,2110 \mathrm{~g} \quad, \quad 0,3606 \mathrm{CO}_{2}, 0,0424 \mathrm{H}_{2} \mathrm{O}$.

$0,2103 \mathrm{~g} \quad, \quad 0,1030 \mathrm{AgBr}$.

$0,6474 \mathrm{~g}, 0,316 \mathrm{AgBr}$.

\begin{tabular}{lcrr} 
& Ber. für $\mathrm{C}_{10} \mathrm{H}_{8} \mathrm{BrO}_{8}$ & \multicolumn{2}{c}{ Gef. } \\
$\mathrm{C}$ & 46,94 & 46,72 & 46,61 \\
$\mathrm{H}$ & 2,20 & 2,20 & 2,25 \\
$\mathrm{Br}$ & 19,55 & 20,68 & 20,85
\end{tabular}


Die zu hohen Bromwerte rühren wahrscheinlich von einer geringen Beimengung eines höher bromierten Produktes her.

Ein saures Kaliumsalz von der Zusammensetzung $\mathrm{C}_{16} \mathrm{H}_{8} \mathrm{O}_{8} \mathrm{BrK}+\mathrm{C}_{16} \mathrm{H}_{9} \mathrm{O}_{8} \mathrm{Br}$ erhält man, wenn man $1 \mathrm{~g}$ Monobromcoccin mit einer Lösung von $3 \mathrm{~g}$ Kaliumacetat in $30 \mathrm{ccm} W$ asser übergießt und mit $160 \mathrm{ccm}$ Eisessig kocht, bis eine fast klare Lösung entsteht. Aus der filtrierten Lösung scheidet es sich beim Erkalten in braunen, zu Büscheln vereinigten Nadeln ab, die mit Wasser und dann mit Essigester ausgewaschen werden.

$0,2414 \mathrm{~g}$ gaben $0,0226 \mathrm{~K}_{\mathbf{8}} \mathrm{SO}_{4}$.

$0,2604 \mathrm{~g} \quad 0,0254 \mathrm{~K}_{2} \mathrm{SO}_{4}$.

Ber.

Gef.

K $\quad 4,57 \quad 4,21 \quad 4,38$

Monobromcoccin vermag im Maximum 5 Äquivalente Baryt zu binden. Die Bestimmung wurde in derselben Weise ausgeführt, wie es bei der Kermessäure beschrieben wurde.

$0,0465 \mathrm{~g}$ verbrauchten $5,9 \mathrm{ccm} \mathrm{n/10-Barytwasser.} \mathrm{Berechnet}$ $5,7 \mathrm{ccm}$.

Acetylverbindung. Man gibt zu $1 \mathrm{~g}$ Monobromcoccin $25 \mathrm{ccm}$ Essigsäureanhydrid und einen Tropfen konz. Schwefelsäure, worauf rasch klare Lösung eintritt. Nach längerem Stehen gießt man auf Eis nnd krystallisiert die Acetylverbindung aus Eisessig um. Gelbe Krystalle, die sich in Natriumbicarbonat mit gelber Farbe lösen and durch Natronlauge verseift werden.

$0,2445 \mathrm{~g}$ gaben $0,4426 \mathrm{CO}_{2}$ und $0,0685 \mathrm{H}_{2} \mathrm{O}$.

$\begin{array}{lcc}\text { Ber. für } \mathrm{C}_{10} \mathrm{H}_{5} \mathrm{O}_{8} \mathrm{Br}\left(\mathrm{COCH}_{3}\right)_{4} & \text { Gef. } \\ \mathrm{C} & 49,92 & 49,37 \\ \mathrm{H} & 2,97 & 3,13\end{array}$

Die Acetylbestimmung wurde in üblicher Weise ausgeführt. Man verseifte mit kohlensäurefreier Natronlauge und destillierte die Essigsäure nach dem Ansänern mit Phosphorsäure ab.

$0,2969 \mathrm{~g}$ verbrauchten $20,4 \mathrm{cem} \approx / 10^{-K a l i l a u g e . ~ B e r e e h n e t ~ f u ̈ r ~}$ vier Acetylgruppen $20,6 \mathrm{ccm}$. 
Oxydation des Monobromcoccins.

Oxydiert man Monobromeoccin in alkoholischer Lösung mit Wasserstoffsuperoxyd und einer Spur Manganosalz als Katalysator in der Wärme, so fallen beim Ansäuern gelbe Krystalle in kleiner Menge ans, die noch nicht untersucht wurden, das Filtrat enthält Cochenillesäure, die man durch Ausäthern isolierte und durch Krystallisation reinigte. Sie ist durch den Schmelzpunkt, die Eisenchloridreaktion und das charakteristische Bariumsalz leicht zu erkennen.

\section{Tribromcoccin.}

Tribromcoccin läßt sich sowohl aus Monobromcoccin. wie auch direkt aus Kermessäure erhalten, wenn man in absolut methylalkoholischer Lösung bromiert. In beiden Fällen ließ sich Entwicklung von Kohlendioxyd nachweisen. $1 \mathrm{~g}$ Monobromcoccin wird in $20 \mathrm{ccm}$ wasserfreiem Methylalkobol gelöst und mit $2 \mathrm{ccm}$ Brom versetzt. Nach längerem Stehen scheidet sich ein Nebenprodukt, das vier Bromatome im Molekül enthält und von dem später die Rede sein wird, in sehr schönen gelben Krystallen ab. Die Ausbente an diesem ist, ohne dab der Grund anzugeben wäre, bei anscheinend ganz gleichen Versuchsbedingungen variabel, sie beträgt meist etwa $0,1 \mathrm{~g}$, steigt aber zuweilen bis $0,4 \mathrm{~g}$. Man saugt die Krystalle ab und gibt zur Lösung $15-20 \mathrm{ccm}$ konz. wäbrige Bromwasserstoffsäure. Die braungelbe Farbe schlägt sofort in rot um und es scheiden sich rote Krystalle von Tribromcoccin in reichlicher Menge, 0,8-0,9 g, ab.

Ganz ähnlich ist die Darstellung ans Kermessäure, nur ist es dann nötig, in der Siedehitze zu bromieren. $\mathrm{Zu}$ einer Lösung von $1 \mathrm{~g}$ Kermessäure in Methylalkohol gibt man $2 \mathrm{ccm}$ Brom und kocht etwa eine Minute anf dem Wasserbade. Beim Erkalten erhält man ebenfalls eine Krystallisation der Tetrabromverbindung, das Filtrat gibt mit Bromwasserstoffsäure Tribromcoccin. Die Mengenverhältnisse an beiden Substanzen sind etwa dieselben, 
wie wenn man vom Monobromcoccin ausgeht. Tribromcoccin bildet, aus Eisessig umkrystallisiert, rote, lange, verfilzte Nadeln von prachtvollem Glanz, zuweilen derbere, dunklere Krystalle. Beim Stehen an der Luft werden die Krystalle matt, indem sie Eisessig verlieren. Zugleich wird die Farbe gelbstichiger.

Tribromcoccin ist unlöslich in Wasser, schwer löslich in Eisessig, leicht in Alkohol. Die bei $100^{\circ} \mathrm{im}$ Vakuum getrocknete Substanz schmilzt bei $245-248^{\circ}$ unter Zersetzung. Der Gewichtsverlust beim Trocknen entspricht einem Molekül Eisessig.

$0,5360 \mathrm{~g}$ verloren 0,05̃66 g entsprechend 10,56 Proz. Berechnet 10,29 Proz.

$0,2498 \mathrm{~g}$ gaben $0,3141 \mathrm{CO}_{2}$ und $0,0305 \mathrm{H}_{2} \mathrm{O}$.

$0,2612 \mathrm{~g} " 0,3290 \mathrm{CO}_{2}, 0,0366 \mathrm{H}_{2} \mathrm{O}$.

$0,2392 \mathrm{~g} \quad, \quad 0,2583 \mathrm{AgBr}$.

$0,1040 \mathrm{~g} \quad, \quad 0,1117 \mathrm{AgBr}$.

$0,2199 \mathrm{~g}, \quad$ in 31,56 Phenol $\Delta 0,090^{\circ}$. $0,2004 \mathrm{~g} \quad " 36,38 \quad " \Delta 0,074^{\circ}$. Ber. für $\mathrm{C}_{15} \mathrm{H}_{7} \mathrm{O}_{6} \mathrm{Br}_{3} \quad$ Gef.

$\begin{array}{lcrc}\mathrm{C} & \mathbf{3 4 , 4 2} & 34,29 & 34,35 \\ \mathrm{H} & 1,35 & 1,36 & 1,43 \\ \mathrm{Br} & 45,87 & 45,95 & 45,75 \\ \mathrm{M} & 523 & 557 & 536\end{array}$

Tribromcoccin löst sich in Natronlauge mit viel mehr blaustichiger Farbe als die Monobromverbindung; am Absorptionsspektrum zeigt sich das dadurch, dab die Banden etwas gegen das rote Ende $z u$ verschoben sind. Die alkalische Lösung sowohl von Kermessäure, wie von Mono- und Tribromcoccin wird an der Luft entfärbt, ähnlich wie Purpurinlösungen; beim Tribromcoccin verläuft jedoch diese Autoxydation viel rascher wie bei den anderen Farbstoffen. In Natriumacetatlösung ist Monobromcoccin löslich, Tribromcoccin löst sich erst auf, scheidet aber alsbald ein schwer lösliches blauviolettes Natriumsalz ab.

In konz. Schwefelsäure löst sich Tribromeocein mit rotvioletter Farbe, die auf Zusatz von Borsäure in tiefblau umschlägt. 
Die rote Lösung von Tribromcoccin in Methylalkohol wird auf Zusatz von Brom braungelb, aus dieser Lösung fällt mit Wasser ein gelber, flockiger Niederschlag aus, der nicht näher untersucht wurde. Auf Zusatz von Bromwasserstoff jedoch wird die Farbe der methylalkoholischen Lösung sofort wieder rot und unverändertes Tribromeoccin krystallisiert aus. Offenbar enthält die braungelbe Lösung ein leicht zerlegbares Bromadditionsprodukt, und diese Substanz ist auch in den Lösungen vorhanden, die bei der Bromierung von Monobromcoccin und von Kermessäure entstehen.

Tribromeoccin vermag im Maximum vier Äquivalente Baryt zu binden.

$0,1952 \mathrm{~g}$ verhrauchten $15,3 \mathrm{cem} \mathrm{n} / 10^{-B a r y t w a s s e r}$. Berechnet $14,9 \mathrm{~cm}$.

Es bildet eine Tetraacetylverbindung. Man erhält sie, wenn man Tribromcoccin mit Essigsäureanhydrid und einem Tropfen Schwefelsäure längere Zeit stehen läßt. Die Substanz geht langsam in Lösung, allmählich scheidet sich ein Teil der Acetylverbindung in grünstichig gelben, verfilzten Nädelchen ab, der Rest wird durch Eingießen in Wasser erhalten. Sie läbt sich aus Eisessig umkrystallisieren, Schmelzp. $223^{\circ}$. Unlöslich in Bicarbonat, durch Natronlauge bei längerem Stehen oder beim Erwärmen verseifbar.

Da die prozentuale Zusammensetzung sich für jede Acetylgruppe im Kohlenstoffgehalt um 1 Proz., im Bromgehalt um 2 Proz. ändert, läßt sich in diesem Fall die Anzahl der Acetylgruppen aus der Elementaranalyse erkennen.

$0,1807 \mathrm{~g}$ gaben $0,2657 \quad \mathrm{CO}_{2}$ und $0,0382 \mathrm{H}_{2} \mathrm{O}$.

$0,2088 \mathrm{~g}, \quad 0,1722 \mathrm{AgBr}$.

$\begin{array}{lrr}\text { Ber. für } \mathrm{C}_{15} \mathrm{H}_{3} \mathrm{O}_{6} \mathrm{Br}_{3}\left(\mathrm{COCH}_{3}\right)_{4} & \text { Gef. } \\ \mathrm{C} & 39,94 & 40,10 \\ \mathrm{H} & 2,19 & 2,36 \\ \mathrm{Br} & \mathbf{3 4 , 7 1} & 35,09\end{array}$

Durch rauchende Salpetersäure wird Tribromcoccin zu Nitrococcussäure, 
<smiles>CC1C([N+](=O)[O-])C(O)C([N+](=O)[O-])C(C(=O)O)C1[N+](=O)[O-]</smiles>

oxydiert. Es werden also die Bromatome durch Nitrogruppen verdrängt, wie auch aus Tribromphenol mit Salpetersäure Bromdinitrophenol gebildet wird. ${ }^{1}$ )

Nebenprodukt bei der Darstellung des Tribromcoccins: Es wurden die gelben Krystalle erwähnt, die bei der Darstellung des Tribromcoccins in kleiner, aber wechselnder Ausbeute stets ausfallen. Die Substanz krystallisiert aus Eisessig in gelben glänzenden, meist radial angeordneten Nadeln; sie löst sich in Natronlauge mit himbeerroter, in Schwefelsäure mit rosenroter Farbe, die auf $\mathrm{Zu}$ satz von Borsäure sich wenig nach blau verschiebt. Die Lösungen in Natronlauge und in Schwefelsäure zeigen keine Streifen, sondern allgemeine Absorption vom violetten Ende her. Nach der Analyse kommt dieser Verbindung die Formel $\mathrm{C}_{15} \mathrm{H}_{6} \mathrm{O}_{5} \mathrm{Br}_{4} \mathrm{zu}$; es wäre also ein Bromcoccin, in welchem eine OH-Gruppe durch Brom ersetzt ist. Damit stimmt auch überein, daß sie nur drei Äquivalente Baryt zu binden vermag.

Die Substanz wurde zur Analyse bei $100^{\circ}$ im Vakuum getrocknet.

$$
\begin{aligned}
& 0,2727 \mathrm{~g} \text { gaben } 0,3028 \mathrm{CO}_{4} \text { und } 0,0285 \mathrm{H}_{2} \mathrm{O} \text {. } \\
& 0,1699 \mathrm{~g} \quad, \quad 0,1885 \mathrm{CO}_{2} \quad, \quad 0,0193 \mathrm{H}_{2} \mathrm{O} \text {. } \\
& 0,1526 \mathrm{~g} \quad, \quad 0,1724 \mathrm{CO}_{2} \quad, \quad 0,0197 \mathrm{H}_{2} \mathrm{O} \text {. } \\
& 0,2103 \mathrm{~g} \quad, \quad 0,2703 \mathrm{AgBr} \text {. } \\
& 0,1826 \mathrm{~g} \quad, \quad 0,2353 \mathrm{AgBr} \text {. }
\end{aligned}
$$

\begin{tabular}{lcrrr} 
& Ber. für $\mathrm{C}_{15} \mathrm{H}_{6} \mathrm{O}_{5} \mathrm{Br}_{4}$ & \multicolumn{3}{c}{ Gef. } \\
$\mathrm{C}$ & 30,72 & 30,28 & 30,26 & 30,91 \\
$\mathrm{H}$ & 1,03 & 1,17 & 1,27 & 1,45 \\
$\mathrm{Br}^{+}$ & 54,58 & 54,71 & 54,81 &
\end{tabular}

$0,2223 \mathrm{~g}$ verbrauchten $11,7 \mathrm{ccm}{ }^{\mathrm{n}} / 10^{-}$-Barytwasser. Ber. $11,4 \mathrm{ccm}$.

1) Armstrong u. Harrow, Journ. chem. Soc. 29, 477 (1876). 


\section{Zinkstaubdestillation der Kermessäure.}

Die Zinkstaubdestillation der Kermessäure verläuft ähnlich, aber immerhin ein wenig glatter als bei der Carminsäure, man erhält neben öligen Substanzen ein Gemenge von Kohlenwasserstoffen, in welchem das $\boldsymbol{u}$ Methylanthracen reichlicher vorhanden ist, so dab es gelang, diesen Kohlenwasserstoff in nahezu reiner Form und das durch Oxydation mit Chromsäure entstehende $\alpha$-Methylanthrachinon in analysenreinem Zustand zu erhalten. Daneben ist vermutlich Anthracen vorhanden, doch verfügten wir nicht über genügend große Mengen, nm dasselbe bis zum richtigen Schmelzpunkt umzukrystallisieren.

Es ist von Wichtigkeit, die Kermessänre mit einer sehr großen Menge Zinkstaub auf das innigste zu mischen. $1,5 \mathrm{~g}$ Farbstoff wurden zuerst mit $15 \mathrm{~g}$ Zinkstaub zusammengerieben und dies Gemenge noch mit $150 \mathrm{~g}$ Zinkstaub verdünnt. Im Verbrennungsrohr legt man noch eine Schicht reinen Zinkstaubes vor und heizt im Wasserstoffstrom rasch an. Bei gut gelungener Operation sammelt sich im vorderen Teile des Rohres ein dicker, rasch erstarrender Tropfen, in die Vorlage geht nur wenig über. Die tusbeute an Rohprodukt beträgt $0,15-0,2 \mathrm{~g}$.

Die halbfeste Masse wird unter Zusatz des gleichen Gewichtes Pikrinsäure in kalt gesättigter äthylalkoholischer Pikrinsäurelösung in der Siedehitze gelöst. Beim Finstellen in Eis krystailisiert das Gemenge der Pikrate iı schönen, scheinbar einheitlichen Prismen von der Farbe und dem Glanz der Chromsäurekrystalle. Man saugt ab und krystallisiert nochmals aus einer möglichst kleinen Menge kalt gesättigter Pikrinsäurelösung um. Die Pikrinsäuremutterlangen enthalten die schmierigen Verunreinigungen; das Pikrat löst man in Alkohol und zerlegt es mit sehr verdünntem Ammoniak. Das Gemenge der Kohlenwasserstoffe, $0,3 \mathrm{~g}$ aus $0,5 \mathrm{~g}$ Rohprodukt, zerlegt man sodann durch Krystallisation aus einer nicht zu großen Menge Äthylalkohol in zwei Teile. 
Die sich beim Erkalten ausscheidenden Blättchen, welche unscharf zwischen $170-180^{\circ}$ schmolzen, wurden noch zweimal aus Eisessig umkrystallisiert. Der Schmelzpunkt liegt dann bei $196^{\circ}$ - Schmelzpunkt des Anthracens $213^{\circ}$.

Durch Oxydation mit Chromsäure erhielt man ein Chinon, das wie Anthrachinon aussieht und wie dieses mit Natriumhydrosulfit eine rote Lösung gibt und dessen Schmelzpunkt nach zweimaligem Umkrystallisieren bei $265^{\circ}$ lag. Vermutlich liegt hier ein noch nicht ganz reines Anthrachinon vor.

Die alkoholische Mutterlange des Anthracens wurde zur Trockne eingedampft, und der Rückstand in einer zur Lösung nicht ganz ausreichenden Menge Methylalkohol aufgenommen. Anf Zusatz von Styphninsäure krystallisieren rote Nädelchen, die noch zweimal aus Methylalkohol umkrystallisiert wurden. Der Schmelzpunkt liegt dann bei $170^{\circ}$.

Die zum Vergleiche hergestellte Styphninsäureverbindung des $\alpha$-Methylanthracens schmilzt bei $177-178^{\circ}$.

Der aus dem Styphnat in Freiheit gesetzte Kohlenwasserstoff wurde aus verdünntem Alkohol umkrystallisiert und schmolz bei $81^{\circ}$, während der Schmelzpunkt des reinen $\alpha$-Nethylanthracens bei $85-86^{\circ}$ liegt.

Schließlich wurde der Kohlenwasserstoff in Eisessiglösung mit Chromsäure oxydiert. Das Chinon krystallisiert aus Eisessig in den äußerst charakteristischen, zu ährenförmigen Aggregaten angeordneten Nädelchen und schmolz bei $170-171^{\circ}$ wie $\alpha$-Methylanthrachinon.

Die Analyse wurde von Herrn Heinze nach der mikrochemischen Methode Pregls ausgeführt.

$3,116 \mathrm{mg}$ gaben $9,235 \mathrm{CO}_{2}$ und $1,265 \mathrm{H}_{2} \mathrm{O}$. $3,877 \mathrm{mg} \quad, \quad 11,53 \quad \mathrm{CO}_{2} \quad, \quad 1,610 \mathrm{H}_{2} \mathrm{O}$.

\begin{tabular}{ccrr} 
& Ber. für $\mathrm{C}_{15} \mathrm{H}_{10} \mathrm{O}_{2}$ & \multicolumn{2}{c}{ Gef. } \\
$\mathrm{C}$ & 81,05 & 80,83 & 81,11 \\
$\mathrm{H}$ & 4,54 & 4,54 & 4,64
\end{tabular}




\section{Anhang: Das Wachs aus Kermes.}

Bearbeitet von A. E. Sherndal.

Bei der Verarbeitung der Droge ${ }^{1}$ ) geht in den ersten ätherischen Extrakt eine sehr reichliche Quantität von Fett and Wachs. Es schien nicht ohne Interesse, dasselbe zu untersuchen, da Liebermann ${ }^{2}$ ) bei der Untersuchung der Cochenille ein Wachs, das Coccerin, aufgefunden hatte, das in seiner Zusammensetzung sehr wesentlich von den gewöhnlichen Wachsarten abweicht; es ist zusammengesetzt aus einer Oxysäure, der Coccerylsäure $\mathrm{C}_{31} \mathrm{H}_{62} \mathrm{O}_{3}$ und einem zweiwertigen Alkohol, dem Coccerylalkshol $\mathrm{C}_{30} \mathrm{H}_{62} \mathrm{O}_{2}$.

Die Trennung der Hauptmenge des Kermeswachses von Fett bereitet keine Schwierigkeit, da es aus der Ätherlösung auskrystallisiert, während das Fett leichter löslich ist. Man digeriert es dann nochmals mit Aceton und krystallisiert schließlich mehrfach aus Chloroform um und erhält es auf diese Weise ganz rein in weißen glänzenden Blättchen, die nach dem Verdunsten des Krystallchloroforms bei $81^{\circ}$ schmelzen.

$0,2515 \mathrm{~g}$ gaben $0,7540 \mathrm{CO}_{9}$ und $0,3086 \mathrm{H}_{2} \mathrm{O}$.

$\begin{array}{ccc} & \text { Ber. für } \mathrm{C}_{52} \mathrm{H}_{104} \mathrm{O}_{2} & \text { Gef. } \\ \mathrm{C} & 82,02 & 81,76 \\ \mathrm{H} & 13,78 & 13,76\end{array}$

Da bei der Größe des Moleküles die Analyse allein keine Entscheidung über die Zusammensetzung geben kann, wurde das Wachs verseift und die Säure sowie der Alkohol isoliert.

Man verseift durch Kochen mit alkoholischer Natronlauge und giebt in gesättigte Kochsalzlösung, wäscht die Masse, welche den freien Alkohol und das Natriumsalz der Säure enthält, mit Kochsalzlösung aus und trocknet sie unter Zusatz von ein wenig Natronlauge - um

3) Ber. d. d. chem. Ges. 43, 1387 (1910).

2) Ber. d. d. chem. Ges. 18, 1975 (1885); 19, 328 (1886); 20, 959 (1887). 
Hydrolyse za vermeiden - auf dem Dampfbad und zuletzt im Exsiccator. Das trockne Pulver wurde im Soxhletapparat erschöpfend mit Äther extrahiert.

Der Alkohol krystallisiert aus Äther in schönen weißen Blättchen, die bei $76^{\circ}$ schmelzen; er ist leicht löslich in Alkohol, Äther und Aceton. Es zeigte sich, das der Alkohol nichts anderes ist als Cerylalkohol.

$0,2446 \mathrm{~g}$ gaben $0,7289 \mathrm{CO}_{2}$ und $0,3027 \mathrm{H}_{2} \mathrm{O}$.

$\begin{array}{ccc} & \text { Ber. für } \mathrm{C}_{\mathbf{2 6}} \mathrm{H}_{54} \mathrm{O} & \text { Gef. } \\ \mathrm{C} & 81,59 & 81,27 \\ \mathrm{H} & 14,23 & \mathbf{1 3 , 8 5}\end{array}$

Zar Feststellung der Identität wurde noch das bei $63-64^{0}$ schmelzende Acetat ${ }^{1}$ ) hergestellt, sowie nach der Methode von $\mathrm{Hell}^{2}$ ) die Wasserstoffmenge bestimmt, welche in Freiheit gesetzt wird, wenn ein primärer Alkohol durch Erhitzen mit Natronkalk in die Säure verwandelt wird.

Die Fettsäure wurde aus dem Rückstand der Ätherextraktion mit Salzsäure in Freiheit gesetzt. Sie erwies sich als Cerotinsäure. Der Schmelzpunkt der aus Benzin umkrystallisierten Substanz lag bei $78-79^{\circ}$.

$0,2376 \mathrm{~g}$ gaben $0,6852 \mathrm{CO}_{2}$ und $0,2829 \mathrm{H}_{2} \mathrm{O}$.

$\begin{array}{ccc} & \text { Ber. für } \mathrm{C}_{28} \mathrm{H}_{52} \mathrm{O}_{2} & \text { Gef. } \\ \mathrm{C} & 78,71 & 78,65 \\ \mathrm{H} & 13,22 & 13,32\end{array}$

Auch die Titration mit alkoholischer Kalilauge gab Werte, die gut auf Cerotinsäure stimmten.

Demnach ist das Wachs Cerotinsäurecerylester. Eben dieser Ester findet sich auch im sogenannten chinesischen Insektenwachs, das von einer Schildlausart Coccus ceriferus abgeschieden wird. Neuerdings liegt über denselben eine Untersuchung von Lipp und $K \mathfrak{a h n}{ }^{3}$ ) vor.

Das Fett des Kermes ist ein Glycerinfettsäureester; eine Untersuchung desselben bot kein besonderes Interesse.

1) Henriques, Ber. d. d. chem. Ges. 30, 1415. (1887).

2) Diese Annalen 223, 269 (1884).

3) Journ. f. prakt. Chem. 86, 184 (1912). 